\title{
Hall Thruster and VASIMR VX-100 Force Measurements Using A Plasma Momentum Flux Sensor
}

\author{
Benjamin W. Longmier ${ }^{1}$ \\ University of Houston, Department of Physics, Houston, TX 77204, USA \\ Edgar A. Bering, III $^{2}$ \\ University of Houston, Departments of Physics and ECE, Houston, TX 77204, USA \\ Bryan M. Reid ${ }^{3}$, Alec D. Gallimore ${ }^{4}$ \\ University of Michigan, Department of Aerospace Engineering, Ann Arbor, MI 48109 USA \\ Jared P. Squire ${ }^{5}$, Tim W. Glover ${ }^{6}$, Franklin R. Chang-Díaz ${ }^{7}$ \\ Ad Astra Rocket Company, Webster, TX 77598, USA \\ and \\ Michael Brukardt ${ }^{8}$ \\ University of Houston, Department of Physics, Houston, TX 77204, USA
}

\begin{abstract}
The accuracy of a plasma impact force sensor was compared to that of the more commonly used inverted pendulum thrust stand using a $5 \mathrm{~kW}$ Xe Hall effect thruster (HET). An improved plasma momentum flux sensor (PMFS) was designed and constructed based on a previous design. Real-time force measurements were made with both the PMFS and the inverted pendulum thrust stand. The PMFS measured the force exerted onto it from the HET exhaust plume with a resolution of $0.1 \mathrm{mN}$, and an average discrepancy of $2 \%$ compared to thrust stand measurements. Experiments were completed using a $9 \mathrm{~m}$ by $6 \mathrm{~m}$ cylindrical vacuum chamber. The total force from the HET was modulated from $34 \mathrm{mN}$ to $356 \mathrm{mN}$ by varying both the anode voltage, from $150 \mathrm{~V}$ to $500 \mathrm{~V}$, and the neutral Xe gas flow rate from $5 \mathrm{mg} / \mathrm{s}$ to $15 \mathrm{mg} / \mathrm{s}$. Initial force measurements from the Variable Specific Impulse Magnetoplasma Rocket (VASIMR) VX-100 are analyzed and compared to RPA data.
\end{abstract}

\footnotetext{
${ }^{1}$ Postdoctoral Fellow, Physics Department, 141 W. Bay Area Blvd., Webster, TX 77584, Member

${ }^{2}$ Professor, Physics and ECE, 617 Science \& Research Bldg 1, Houston, TX 77004, Associate Fellow

${ }^{3}$ Ph.D. Candidate, Aerospace Engineering, 1919 Green Rd Room B107, Ann Arbor, MI 48109, Member

${ }^{4}$ Professor of Aerospace Engineering and of Applied Physics, Associate Dean for Academic Programs and Initiatives, Aerospace Engineering, 1919 Green Rd Room B107, Ann Arbor, MI 48109, Associate Fellow

${ }_{6}^{5}$ Director of Research, Ad Astra Rocket Company, 141 W. Bay Area Blvd. Webster, TX 77584, Member

${ }^{6}$ Director of Development, Ad Astra Rocket Company, 141 W. Bay Area Blvd., Webster, TX 77584, Member

${ }^{7}$ CEO, Ad Astra Rocket Company, 141 W. Bay Area Blvd., Webster, TX 77584, Associate Fellow

${ }^{8}$ Graduate Student, Physics Department, 141 W. Bay Area Blvd., Webster, TX 77584
} 


\section{Introduction}

$\mathrm{I}$ $\mathrm{t}$ is often physically or financially impractical to measure the force produced by an electric thruster using the traditional technique of mounting the thruster on an inverted pendulum thrust stand. Instead, a simple, robust, and low-cost plasma impact force sensor can be used to reliably determine the total force produced by an electric propulsion thruster. Until now, only a handful ${ }^{1-5}$ of experiments focused on impact force sensors. Virtually no experiments have attempted to compare the accuracy of an impact thrust sensor to the more commonly used inverted pendulum thrust stand. Experimental validation tests were completed using the P5 thruster $^{6-8}$, a $5 \mathrm{~kW}$ Xe Hall effect thruster (HET), and demonstrated that the thrust from the P5 inferred from measurements made with a plasma impact force sensor (PMFS) agree very closely with the thrust measurements made by a conventional inverted pendulum thrust stand. The same PMFS sensor was then used to take force measurements in the plasma exhaust of the Variable Specific Impulse Magnetoplasma Rocket (VASIMR) VX-100. Preliminary force results are reported from one of the VASIMR test shots. Multiple test shots with RPA data are compared with the PMFS data.

The plasma momentum flux sensor (PMFS) described in this paper was designed and constructed based on a previous design. ${ }^{1}$ The PMFS was placed in the flowing plasma stream of the P5 HET where real time force measurements were made with both the PMFS and the thrust stand. The PMFS was able to measure the force exerted on it with a resolution of $0.1 \mathrm{mN}$, and an average discrepancy of $2 \%$ compared to the thrust stand. The total force from the HET was modulated from $34 \mathrm{mN}$ to $356 \mathrm{mN}$ by varying both the anode voltage, from $150 \mathrm{~V}$ to 500 $\mathrm{V}$, and the neutral Xe gas flow rate from $5 \mathrm{mg} / \mathrm{s}$ to $15 \mathrm{mg} / \mathrm{s}$. Additionally, the majority of the force data taken during the experiment campaign was completed as a 'blind study' where force measurements from both techniques were disclosed only after the experiment was completed. Though the experiments show a high accuracy for HETs with ion energies ranging from $130 \mathrm{eV}$ to $440 \mathrm{eV}$, the PMFS showed no indication of saturation with ion energy, plasma flux, local magnetic field strength, or force magnitude, indicating that the full range of the PMFS usefulness is still largely unexplored. The PMFS may find uses in many flowing plasma and electric propulsion applications where an accurate, low cost force sensor is required.

\section{Experimental Setup}

\section{A. Chamber and diagnostic setup}

The Large Vacuum Test Facility (LVTF), a cylindrical stainless-steel clad vacuum chamber at the University of Michigan-Ann Arbor, is a 9-meter-long by 6-meter-diameter chamber used primarily for the testing of research and space qualified electric propulsion thrusters. ${ }^{9}$ The chamber is evacuated by two $2,000 \mathrm{cfm}$ blowers and four 400 cfm mechanical pumps to a rough vacuum level of 30 to 100 mTorr. ${ }^{9}$ To reach high vacuum, $\sim 10^{-7}$ Torr, the vacuum chamber employs seven CVI TM-1200 nude cryopumps, with a combined pumping speed of 500,000 1/s of air, and 240,000 1/s of xenon. However, because of the low flow rates of the P5 thruster, only 4 of the 7 cryopumps were used during this experiment campaign with a resulting Xe pumping speed of 140,000 1/s. The 4 cryopumps yielded an average base vacuum pressure of $3 \times 10^{-7}$ Torr, and a chamber pressure of $4.8 \times 10^{-6}$ Torr, $7.9 \times 10^{-6}$ Torr, and $1.1 \times 10^{-5}$ Torr with a combined thruster and cathode flow rate of $5 \mathrm{mg} / \mathrm{s}, 10 \mathrm{mg} / \mathrm{s}$, and $15 \mathrm{mg} / \mathrm{s} \mathrm{Xe}$ respectively.

The P5 thruster was mounted on an inverted pendulum thrust stand, described in detail by Haag et al. ${ }^{10}$. The inverted pendulum thrust stand, and hence the P5 thruster, was fixed in place within the vacuum chamber, and the PMFS was mounted on a 2-axis translation stage within the vacuum chamber. The translation stage allowed the PMFS to scan a separation distance of 94 to $23 \mathrm{~cm}$ from the thruster exit plane, and allowed for a lateral range of $100 \mathrm{~cm}: 70 \mathrm{~cm}$ from the axis in one direction and $30 \mathrm{~cm}$ from the axis in the other direction. For all force measurements the graphite PMFS target was centered radially and placed at various axial separation distances. However, the translation stage moved through its entire lateral range of motion in order to obtain a radial ion flux profile with a Faraday probe biased into ion saturation. A 3-millimeter-diameter tungsten rod enclosed in an alumina shroud served as the Faraday probe. The tip of the rod was flush with the alumina tube in order to help prevent a radial sheath expansion with biases more negative than the floating potential. The Faraday probe was mounted at the same vertical and axial position as the graphite PMFS target, but with a $20 \mathrm{~cm}$ radial offset from the PMFS target. The surface of the Faraday probe was also cleaned for 10 minutes via xenon ion bombardment prior to data collection.

In order to measure the ion energy in the plume, a Retarding Potential Analyzer (RPA) was installed on a 60centimeter-long boom that was able to sweep through the plume. ${ }^{11}$ Measurements were then taken with the RPA 
located $58.5 \mathrm{~cm}$ from the exit plane of the thruster. The RPA was mounted on the boom structure so that it could be moved out of the thruster plume when PMFS measurements were taken. A small floating probe was also installed on the boom and was used to measure the plasma floating potential $58.5 \mathrm{~cm}$ from the exit plane of the thruster.

\section{B. Hall Thruster}

All data within this paper, with the exception of Section III D, were taken using the P5 Xe HET, a 5-kW HET designed and used primarily for research purposes. ${ }^{5-8}$ Previous data indicate that the P5 has performance characteristics comparable to commercial space-qualified 5-kW HETs. ${ }^{5-8}$ The P5 has a discharge chamber outer diameter of $173 \mathrm{~mm}$ and channel width of $25 \mathrm{~mm}$. Although designed for steady-state operation at $5 \mathrm{~kW}$, the thruster was operated at power levels from $0.71 \mathrm{~kW}(150 \mathrm{~V}, 4.70 \mathrm{~A})$ to $7.65 \mathrm{~kW}(500 \mathrm{~V}, 15.30 \mathrm{~A})$ during this experiment. The P5 used xenon gas as the propellant for the duration of this testing campaign and for all data presented in this paper.

\section{VASIMR VX-100}

The VAriable Specific Impulse Magnetoplasma Rocket (VASIMR ${ }^{\mathrm{TM}}$ ), Fig. 1, is a high power, radio frequency-driven magnetoplasma rocket, capable of $I_{s p} /$ thrust modulation at constant power. The physics and engineering of this device have been under study since 1980 . The plasma is produced by an integrated helicon discharge. The bulk of the plasma energy is added in a separate downstream stage by ion cyclotron region heating (ICRH). Axial momentum is obtained by the adiabatic expansion of the plasma in a magnetic nozzle. Thrust/specific impulse ratio control in the VASIMR ${ }^{\mathrm{TM}}$ is primarily achieved by the selective partitioning of the RF power to the helicon and ICRH systems, with the proper adjustment of the propellant flow. However, other complementary techniques are also being considered. A NASA-led, research effort, involving several teams in the United States, continues to explore the scientific and technological foundations of this concept. The research is multifaceted and involves theory, experiment, engineering design, mission analysis, and technology development. Experimentally, high-density, stable plasma discharges have been generated in Helium, Hydrogen, Deuterium, Argon and Xenon. Ion dynamics in the exhaust are studied using a variety of probes, gridded energy analyzers (RPA's), microwave interferometry and optical techniques.

The VASIMR ${ }^{\mathrm{TM}}$ engine has three major subsystems, the injection stage, the heating stage and the nozzle as seen in Fig. 2. The use of a separate injection system has allowed us to

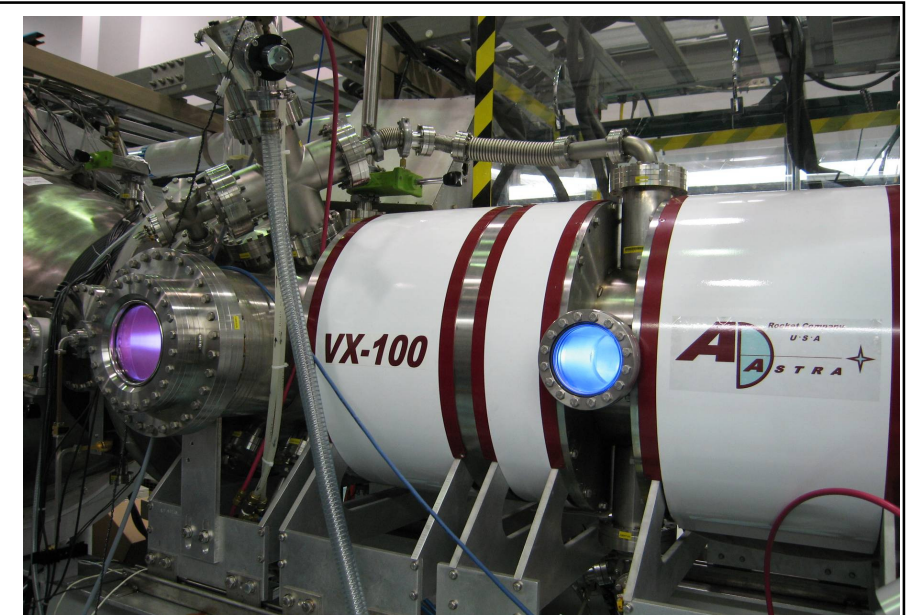

Figure 1. Photograph of the VASIMR VX-100 device. Courtesy of Ad Astra Rocket Company.

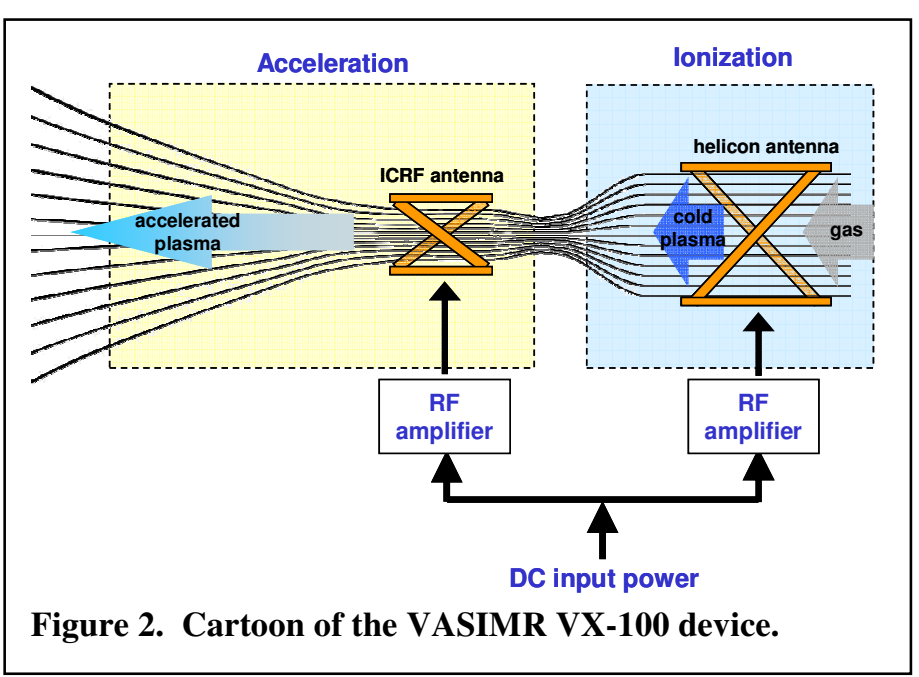
optimize our system for maximum power efficiency over a wide range of gas flow rates. At present, the best choice appears to be a helicon discharge. The next stage downstream is the heating system. Energy is fed to the system in the form of a circularly polarized RF signal tuned to the ion cyclotron frequency. ICRH heating has been chosen because it transfers energy directly and solely to the ions, which maximizes the efficiency of the engine. The system also features a two-stage magnetic nozzle, which accelerates the plasma particles by converting their azimuthal 
energy into directed momentum. The detachment of the plume from the field takes place mainly by the loss of adiabaticity and the rapid increase of the local plasma beta, defined as the local ratio of the plasma pressure to the magnetic pressure.

The VX-100 has demonstrated ICRH antenna efficiency $>90 \%$ and a total coupling efficiency of $\sim 75 \%$. The rocket performance parameters inferred by integrating the moments of the ion energy distribution corresponds to a thrust of $2 \mathrm{~N}$ at an exhaust velocity of $20 \mathrm{~km} / \mathrm{s}$ with the VX-100 device.

The force data presented in this paper used VX-100 settings of: $25 \mathrm{~kW}$ of RF power to the Helicon stage, $13 \mathrm{~kW}$ of RF power to the ICRH stage. These settings resulted in roughly $50 \mathrm{eV}$ Ar ions in the exhaust region of the plasma flow. The PMFS was mounted roughly $50 \mathrm{~cm}$ downstream of the VX-100 exhaust exit plane.

\section{Concept and Construction of the PMFS}

The PMFS consists of a 9-centimeterdiameter graphite target disc attached to a 10centimeter-long insulating alumina rod. The stiff alumina rod then connects to a small titanium bar $(5.72 \mathrm{~cm} \times 1.30 \mathrm{~cm})$ where a series of 4 high output semiconductor strain gauges, Micron Instruments model number SS-090060-1150P, are mounted between two holes on an "isthmus" on the titanium bar, as seen in Fig. 3. The isthmus acts as a stress concentrator and increases the sensitivity of the device. The strain gauges are connected electrically in a Wheatstone bridge configuration so that changes in temperature of the titanium bar do not affect the linearity of the strain gauge output.

When the electrically floating graphite disc is immersed in flowing plasma (e.g. the plume of a HET) the force from the plasma impacting the graphite target is translated into a strain in the titanium beam through a moment arm equal to the length of the alumina rod plus the clamp length. A small graphite shield was also used to keep the entire titanium bar and strain gauge assembly shielded from the flowing plasma, and associated thermal and electrical noise.

The resolutions of the PMFS and the inverted pendulum thrust stand described in this paper were $0.1 \mathrm{mN}$ and $0.5 \mathrm{mN}$ respectively, which allowed for sufficiently sensitive measurements of the force applied by the exhaust plasma. The typical error associated with the inverted pendulum thrust stand is $\pm 2 \mathrm{mN}$ for a measured force of $100 \mathrm{mN}$.

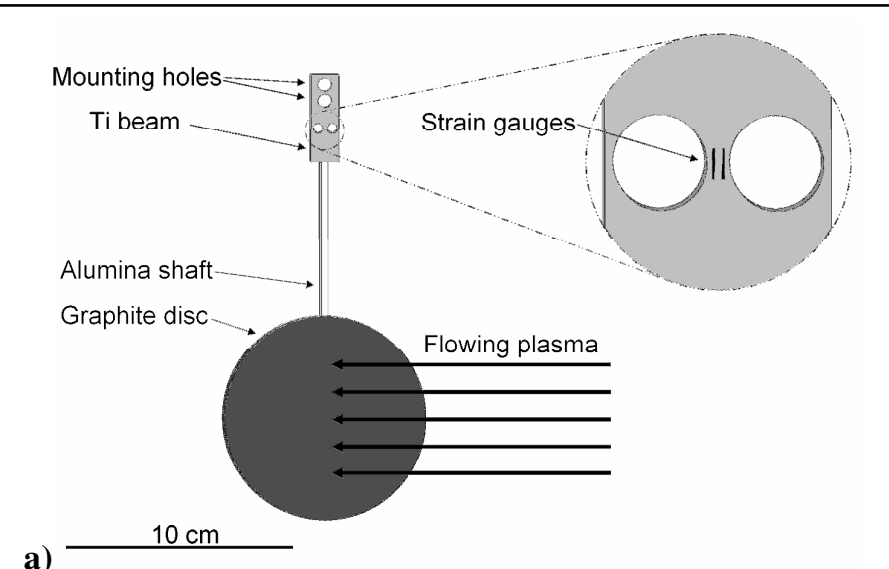

b)

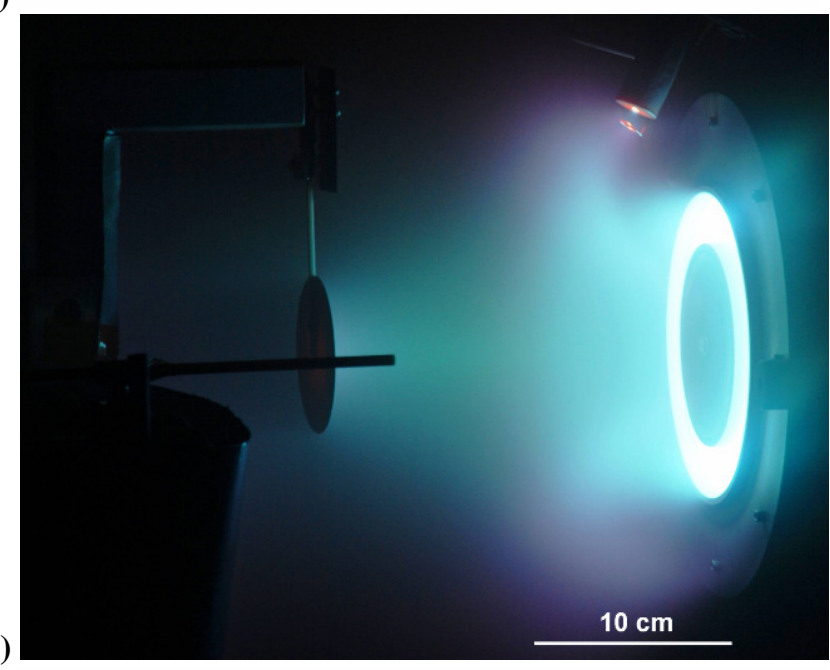

Inverted Pendulum

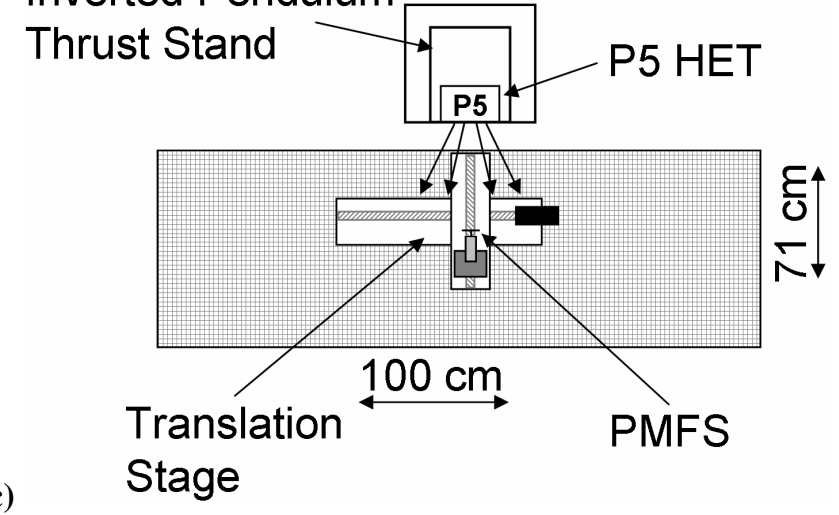

Figure 3. Schematic of the PMFS assembly and zoom in of strain gauge arrangement mounted on the $\mathrm{Ti}$ isthmus, a), and a photo of the P5 HET and PMFS during the closest approach, b). The Faraday probe biased into ion saturation can be seen in the foreground and the PMFS graphite target disc in the background in b). An overhead schematic shows the P5 HET and the force target, c). 
If an increased PMFS force resolution were required, the length of the alumina moment arm could be increased, acting to increase the output from the strain gauges for a particular force applied to the graphite target. However, increasing the arm length of the device also decreases the resonant frequency response of the device. This limitation is generally not a concern for steady-state thruster operation. If the thruster (or some other source of flowing plasma) were operated in a pulsed mode, then data analysis is simplified if the moment arm was selected such that the natural period of the PMFS device is much shorter than the thruster pulse duration.

The graphite target only measured a portion of the total axial force generated by the HET in each measurement. The PMFS target diameter was 52\% of the P5 thruster channel O.D. An azimuthally integrated radial profile of the ion flux was used to account for the portion of the plasma plume that was not intercepted by the graphite target. For each force measurement presented in this paper, a corresponding radial profile of the ion flux was collected by a Faraday Probe and used to determine the total axial force produced by the thruster. Figure 4 is a representative radial ion flux profile from the P5 HET plume; in this case, the graphite target was $50 \mathrm{~cm}$ downstream from the exit plane of the thruster. The ion flux was recorded with a tungsten electrode that was recessed inside of an alumina tube and was biased at $-14.95 \mathrm{~V}$, roughly 3 $T_{e}$ lower in voltage than the plasma potential, but not too low so as to create additional ionization in the far ion saturation region. A high input impedance data logger was used to record the

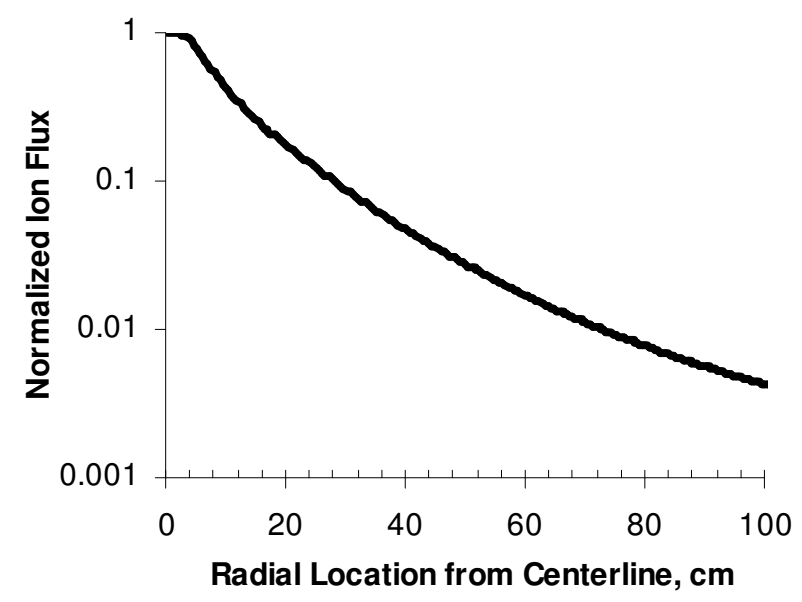

Figure 4. A representative radial profile of the ion flux in the HET plume, $50 \mathrm{~cm}$ downstream from the exit plane of the thruster, with HET parameters of $150 \mathrm{~V}$, and $16.6 \mathrm{~A}, 15 \mathrm{mg} / \mathrm{s} \mathrm{Xe}$. current through a $107.3 \mathrm{ohm}$ shunt resistor

The ratio of the total axial ion flux $(r=0$ to $r=100 \mathrm{~cm}$ ), numerically integrated over the entire plume assuming cylindrical symmetry, to that of the axial ion flux intercepted by the target $(r=0 \mathrm{~cm}$ to $r=4.5 \mathrm{~cm})$ is given by

$$
\frac{\sum_{x=0}^{x=1000} \pi\left(r_{\mathrm{x}+1}^{2}-r_{\mathrm{x}}^{2}\right) I\left(r_{\mathrm{x}}\right)}{\sum_{x=0}^{x=45} \pi\left(r_{\mathrm{x}+1}^{2}-r_{\mathrm{x}}^{2}\right) I\left(r_{\mathrm{x}}\right)}
$$

where $I\left(r_{\mathrm{x}}\right)$ is the ion current as measured by a Faraday probe biased into ion saturation at a radius $r_{\mathrm{x}}$ in the plasma exhaust. Here, $x$ ranges from 0 to 1,000 for $r_{\mathrm{x}}$ values from 0 to $100 \mathrm{~cm}$. Thrust contributions from the plume at radii greater than $100 \mathrm{~cm}$, the maximum range of the translation stage, are negligible for the data presented in this paper.

The total axial force, $F_{\text {Total }}$, produced by the HET is determined by multiplying the force measured by the graphite target, $F_{\text {Target }}$, by Eqn. (1), which becomes

$$
F_{\text {Total }}=F_{\text {Target }} \frac{\sum_{x=0}^{x=1000} \pi\left(r_{\mathrm{x}+1}^{2}-r_{\mathrm{x}}^{2}\right) I\left(r_{\mathrm{x}}\right)}{\sum_{x=0}^{x=45} \pi\left(r_{\mathrm{x}+1}^{2}-r_{\mathrm{x}}^{2}\right) I\left(r_{\mathrm{x}}\right)}
$$


The assumption that the thruster plume is symmetric in the azimuthal direction leads to the largest source of error with the PMFS device. The asymmetries observed in the plume are likely due to non-uniform propellant delivery as the flow rate increased beyond its nominal design point of $10 \mathrm{mg} / \mathrm{s}$. A similar result was reported where fabrication error in the anode resulted in an asymmetric plume at high flow rates. ${ }^{9}$ One way to reduce this error is to construct a 2-D map of the ion flux profile; however, this mapping was not performed owing to the limitations of the translation stage.

Once a total force measurement was numerically integrated from the PMFS measurement and ion flux profile, corrections were made for momentum reflection and sputtering, as described in section IV A.

\section{E. Calibration}

After the PMFS was installed in a rigid location within the vacuum chamber, in this case on a 2 -axis translation stage, and before the vacuum pump down, the PMFS was calibrated in order to find an accurate relationship between the output of the strain gauges and the force applied to the target. Calibration was performed in the same manner as was previously described by Chavers et al. ${ }^{1}$, in which a set of calibrated weights were hung from a tethered string assembly to apply a known tension force on the graphite target disc. Figure 3 shows a representative example of the force-to-voltage relation for the particular device used in this experiment campaign. The strain sensors had an output voltage-to-force relation of $0.64 \pm 0.01 \mathrm{mV} / \mathrm{mN}$ (or $1.56 \mathrm{mN} / \mathrm{mV}$ ). If the PMFS was correctly leveled within the vacuum chamber prior to testing, the resulting force per unit voltage line would intercept the origin, as depicted in Fig. 5.

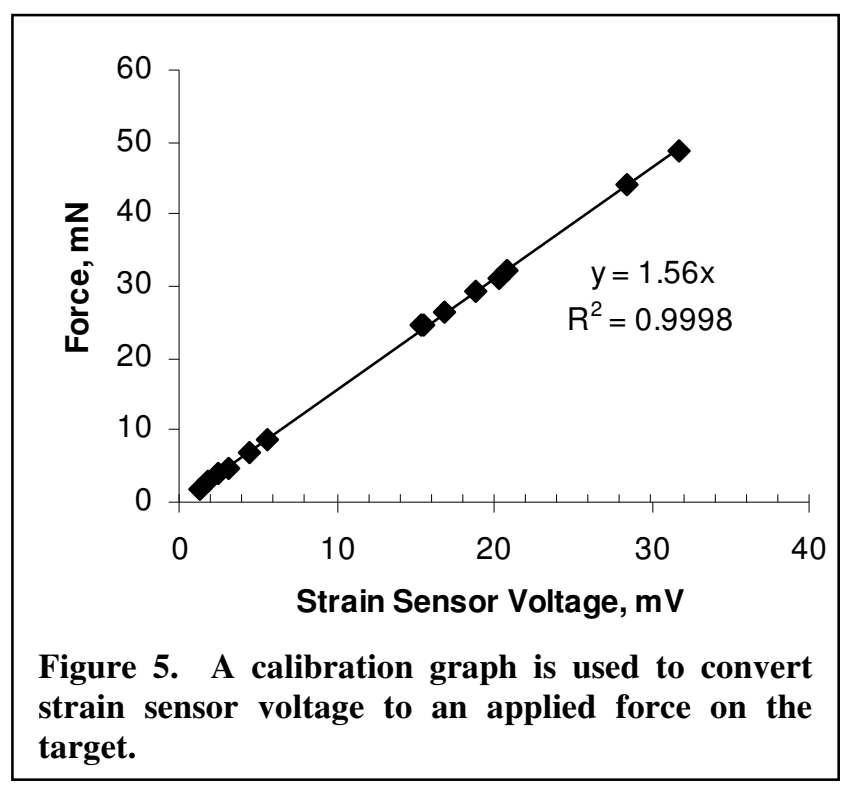

The data from Fig. 5 also indicate that the strain gauges are linear over a force range from 0 to at least $50 \mathrm{mN}$ for this particular device. Because the graphite target did not intercept all of the HET exhaust plume, this translates into a useful measurable P5 thruster force range of at least 0 to $1000 \mathrm{mN}$ for thruster to target separation distances between 23 and $94 \mathrm{~cm}$. A PMFS calibration was performed before and after every vacuum chamber pump down and venting in order to verify that the strain gauges did not exhibit a drift or creep in time. During the PMFS HET experiments, both PMFS calibrations agreed to within $0.3 \%$.

\section{Experimental Demonstration}

\section{A. Thruster-Sensor Separation Distance}

An initial thruster-to-target separation distance experiment was performed in order to determine which separation distances could be tolerated without significantly altering the performance of the thruster. The separation distance was decreased from $94 \mathrm{~cm}$ to $23 \mathrm{~cm}$ in roughly 10-centimeter increments. For each thrust measurement, the thruster was turned on and allowed to stabilize. Then, a radial ion flux profile was taken with the Faraday probe, see Fig. 6, and a relative thrust measurement was taken with both techniques. The thruster was then turned off, and a baseline thrust measurement was taken, also with both techniques. The difference in signal strength between the thrust-on and thrust-off values was used to determine a thruster force. The slope from the respective PMFS and thrust stand calibration curves (i.e. $\mathrm{mN} / \mathrm{mV}$ ) was then used to determine an absolute force value from the relative force measurements. In this way, simultaneous force measurements could be made with both techniques. This procedure also helped to compensate for any drift in thruster performance or force over time. 
An increase in the measured force was registered with both techniques when the PMFS disc approached the HET as seen in Fig 7. The PMFS data presented in Fig. 4 and Table 1 also include corrections for sputtering based on SRIM/TRIM models ${ }^{12}$ (see Section IV A for further sputtering correction details).

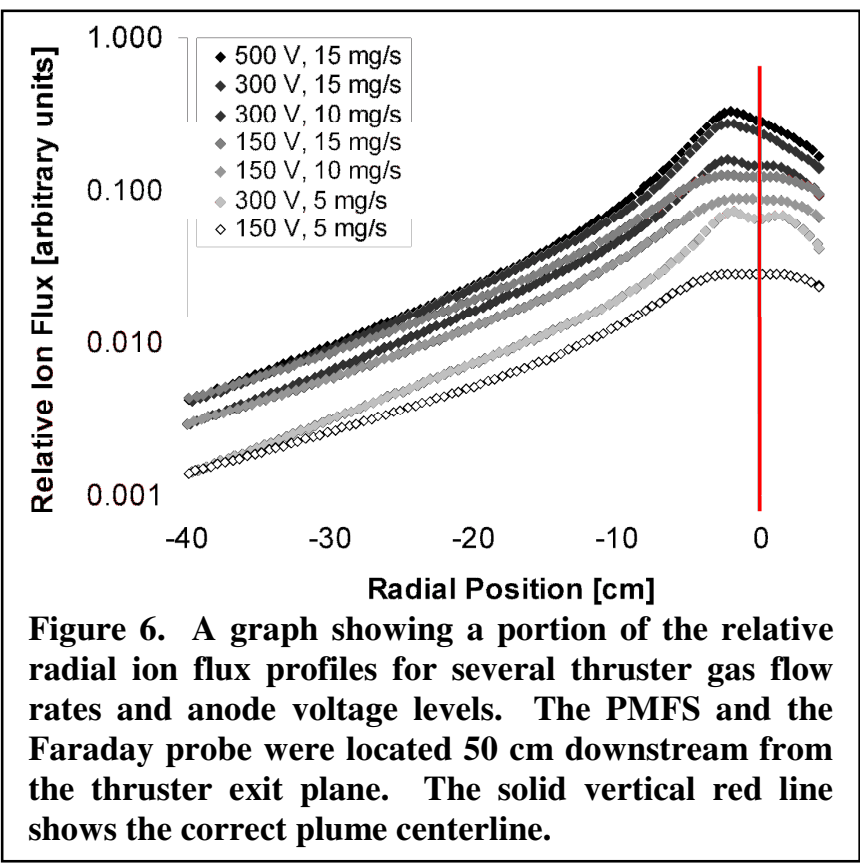

\begin{tabular}{lcccccccc}
\hline \hline Table 1. Measured thrust versus the separation distance of the PMFS target from \\
& \multicolumn{7}{c}{ the HET. } \\
\hline Separation distance, cm & 94 & 80 & 70 & 60 & 50 & 40 & 30 & 23 \\
PMFS, mN & $\mathbf{7 6 . 1}$ & $\mathbf{7 6 . 6}$ & $\mathbf{7 6 . 8}$ & $\mathbf{7 6 . 8}$ & $\mathbf{7 6 . 9}$ & $\mathbf{7 6 . 9}$ & $\mathbf{7 7 . 1}$ & $\mathbf{7 7 . 3}$ \\
Thrust stand, mN & $\mathbf{7 6 . 3}$ & $\mathbf{7 7 . 1}$ & $\mathbf{7 7 . 6}$ & $\mathbf{7 7 . 9}$ & $\mathbf{7 7 . 9}$ & $\mathbf{7 8 . 4}$ & $\mathbf{7 9 . 2}$ & $\mathbf{7 9 . 2}$ \\
Difference, \% & 0.3 & 0.6 & 1.1 & 1.4 & 1.2 & 1.9 & 2.6 & 2.4 \\
Anode Voltage, $\mathrm{V}$ & 300 & 300 & 300 & 300 & 300 & 300 & 300 & 300 \\
Xe flow rate, mg/s & 5 & 5 & 5 & 5 & 5 & 5 & 5 & 5 \\
Anode current, A & 5.1 & 5.1 & 5.1 & 5.1 & 5.1 & 5.1 & 5.1 & 5.1 \\
\hline
\end{tabular}

The increase in thruster force with reduced thrustertarget separation distance was previously observed by Chavers et al. ${ }^{1}$ in a similar experiment (see Section D), and is attributed to an increased neutral pressure near the HET exit plane. Due to finite facility pressure, background neutrals continually reach the thruster discharge channel, become ionized, accelerated, and artificially increase thrust. ${ }^{13}$ As the PMFS approaches the thruster exit plane, the neutral ingestion is expected to increase because of two effects. First, a fraction of the non-ionized neutrals from the thruster will be reflected from the paddle surface with an isotropic distribution. Second, ions will impact the paddle and recombine at the surface to produce an additional isotropic reflected neutral flux. A fraction of these neutralized ions will also reach the thruster and artificially increase the thrust produced by the thruster. The HET was operated at $1.53 \mathrm{~kW}$, producing $5.1 \mathrm{~A}$ of discharge current with an anode bias of $300 \mathrm{~V}$, using a neutral Xe flow rate of $5 \mathrm{mg} / \mathrm{s}$. The average difference between the force

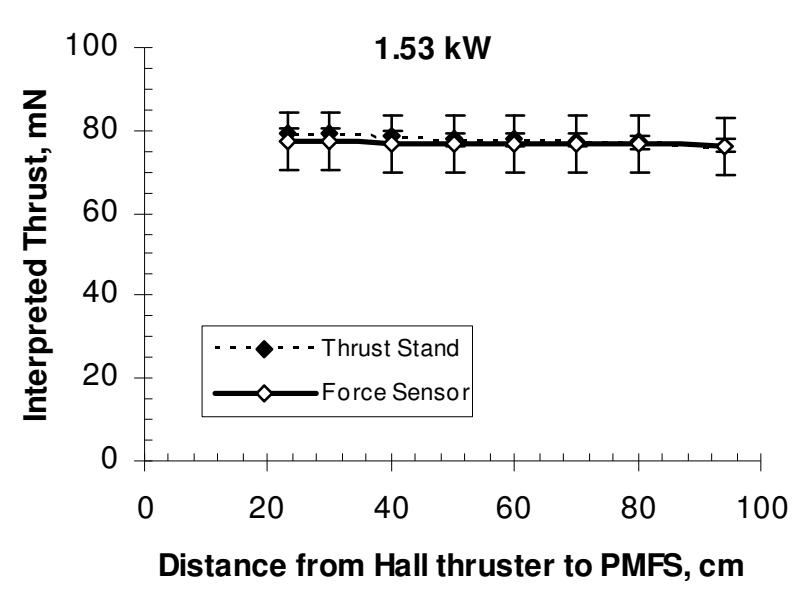

Figure 7. Measured thrust versus the separation distance of the PMFS target and the HET. The thruster was operated at $1.53 \mathrm{~kW}$, using $5 \mathrm{mg} / \mathrm{s} \mathrm{Xe}$, $5.1 \mathrm{~A}$ at $300 \mathrm{~V}$. measurements for the inverted pendulum thrust stand and PMFS techniques is $1.4 \%$ for this data set, which is considerably less than the error associated with either the PMFS or the inverted pendulum thrust stand for this set of experiments. 


\section{Power Variations}

The PMFS was positioned on thruster centerline $50 \mathrm{~cm}$ from the exit plane of the thruster while the Xe flow rate and the thruster anode voltage were altered in order to achieve a range of ion energy, ion flux, and total force. Figure 8 shows the thrust from the thrust stand and the PMFS measurements as a function of the calculated HET power. Table 2 shows the numeric results displayed in Fig. 7. Figure 8 shows a graph of the radial ion flux profiles for several thruster gas flow rates and anode voltage levels with the thruster centerline superimposed on the graph.

The results for the power and are shown in Fig. 8 and Table 2. An accurate centerline for all of the peaks, found by fitting a bimodal distribution to the data in Fig. 6, is located at a radial position of $0 \mathrm{~cm}$ and shown in Fig. 6. The Faraday probe that was mounted on the translation stage had a radial range of $138.1 \mathrm{~cm}$, where the thruster plume centerline located at $0 \mathrm{~cm}$.

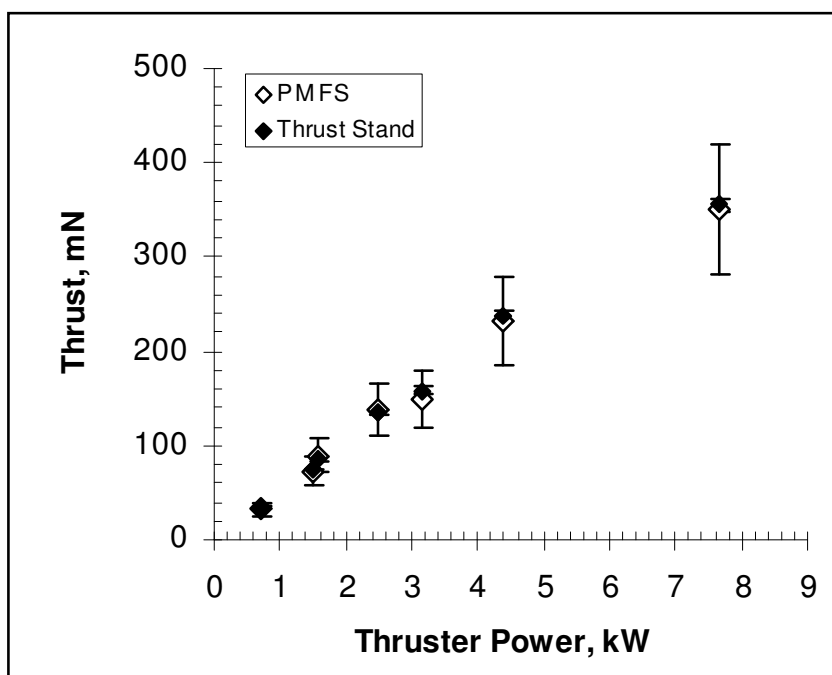

Figure 8. Thrust measurements for a variety of P5 HET power levels. The PMFS was located 50 cm downstream from the thruster exit plane.

\begin{tabular}{lccccccc}
\hline \hline \multicolumn{6}{c}{ Table 2. Measured thrust for a variety of P5 HET power levels. } \\
\multicolumn{6}{c}{ The PMFS was located 50 cm downstream from the thruster exit plane. } \\
\hline Thruster power, kW & 0.71 & 1.50 & 1.58 & 2.49 & 3.15 & 4.38 & 7.65 \\
PMFS, mN & $\mathbf{3 2 . 6}$ & $\mathbf{7 2 . 7}$ & $\mathbf{8 9 . 2}$ & $\mathbf{1 3 8 . 2}$ & $\mathbf{1 4 9 . 7}$ & $\mathbf{2 3 1 . 4}$ & $\mathbf{3 5 0 . 7}$ \\
Thrust stand, mN & $\mathbf{3 3 . 9}$ & $\mathbf{7 3 . 6}$ & $\mathbf{8 5 . 9}$ & $\mathbf{1 3 6 . 6}$ & $\mathbf{1 5 8 . 8}$ & $\mathbf{2 3 7 . 1}$ & $\mathbf{3 5 5 . 8}$ \\
Difference, \% & 3.6 & 1.2 & 3.8 & 1.2 & 5.7 & 2.4 & 1.4 \\
Anode Voltage, V & 150 & 300 & 150 & 150 & 300 & 300 & 500 \\
Xe flow rate, mg/s & 5 & 5 & 10 & 15 & 10 & 15 & 15 \\
Anode current, A & 4.7 & 5.0 & 10.5 & 16.6 & 10.5 & 14.6 & 15.3 \\
\hline
\end{tabular}

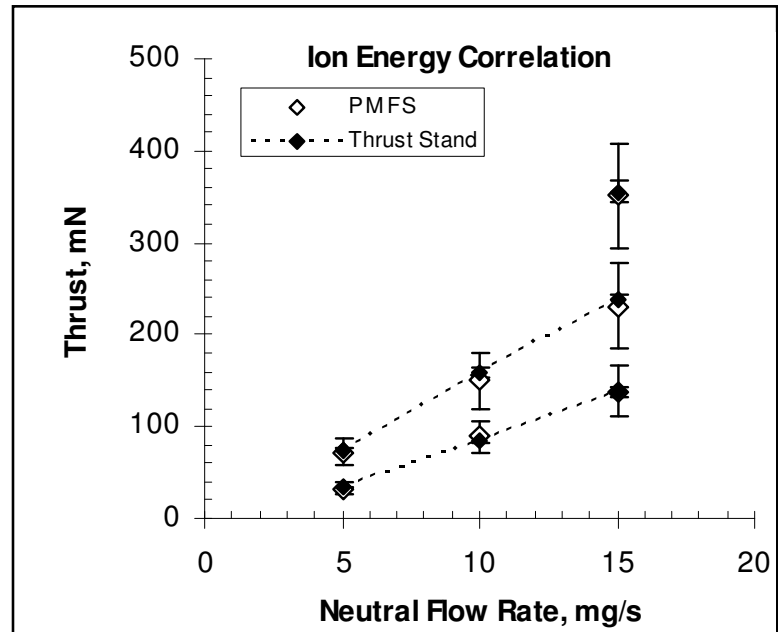

Figure 9. Corrected blind study thrust measurements for a variety of P5 HET power levels. The PMFS was located $50 \mathrm{~cm}$ downstream from the thruster exit plane.

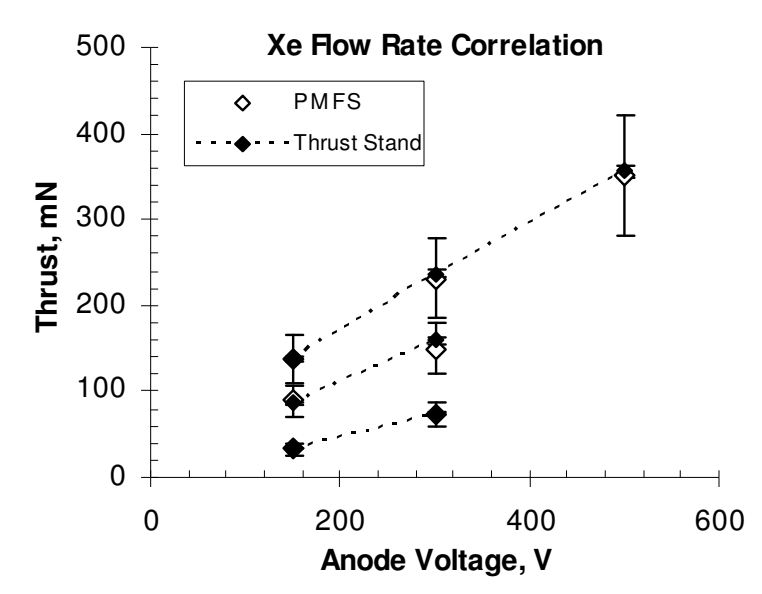

Figure 10. Corrected blind study thrust measurements for a variety of P5 HET power levels. The PMFS was located at $50 \mathrm{~cm}$ downstream from the thruster exit plane.

The largest difference between the thrust stand measurements and the PMFS measurements was 5.7\%, with an average discrepancy for this data set of $2.8 \%$, as seen in Table 3 . 
In an effort to identify a trend in the PMFS accuracy as a function of ion energy and neutral gas flow rate, the relevant PMFS and thrust stand force data are graphed as a function of the thruster neutral flow rate, Fig. 9, and as a function of the thruster anode voltage, Fig. 10. No clear correlation of PMFS accuracy is obvious as the anode voltage or neutral gas flow rate are varied. To first order, the discrepancy between the PMFS and the thrust stand appears to be largely unaffected by the HET system parameters. However, data from Fig. 7 does indicate a discernable trend, with a larger discrepancy between the two force measurement techniques as the PMFS approaches the thruster exit plane from a separation distance of $94 \mathrm{~cm}$ to $23 \mathrm{~cm}$.

The PMFS has proven to be accurate compared to the inverted pendulum thrust stand with a maximum observed difference of 5.7\% over a large range of applied force, ion energy, and neutral gas flow settings. The PMFS should find considerable application in the low-thrust plasma community due to its simplicity, robustness, and verified accuracy.

\section{Initial VASIMR VX-100 Force Data}

The design of the PMFS used for the VX-100 in 2007 experiments allowed for a resolution of the measured force equal to $0.1 \mathrm{mN}$. The natural oscillating period of the device was tuned so that it was much shorter than the pulse duration of each VX-100 shot. That is, the PMFS target would oscillate back and forth 510 times during each plasma shot, as seen in Fig. 11.

The forces from the neutral gas puff, the helicon discharge, and the helicon discharge accelerated by the ICRH boost on the target were $0.7 \mathrm{mN}, 12 \mathrm{mN}$, and $30 \mathrm{mN}$ respectively. Figure 12 shows that a significant amount of force was imparted to the target when only 13 $\mathrm{kW}$ of ICRH power was added to the existing $25 \mathrm{~kW}$ of helicon power. Because the size of the target used in this experiment campaign was significantly smaller than the diameter of the plasma exhaust plume, the target only measured a portion of the total force generated by the VX-100. A radial profile of the ion flux was used to account for the portion of the plasma plume that was not intercepted by the target. The ion flux was measured with a fast reciprocating Langmuir probe biased into ion saturation.

When the full plasma profile is integrated over an azimuthal rotation and corrected for reflected neutral gas and the momentum change from sputtered material, the total force produced by the shot was $810 \mathrm{mN}$.

The PMFS force data is compared to the RPA ion velocity and ion flux data, where the force from the VX-100 is calculated from $F=\dot{m} v$. Figure 13 shows two PMFS force data points superimposed on a graph of the calculated force from RPA and ion flux measurements as a function of ICRH RF power from 0 to $26 \mathrm{~kW}$. The RF helicon power is held fixed at $25 \mathrm{~kW}$.

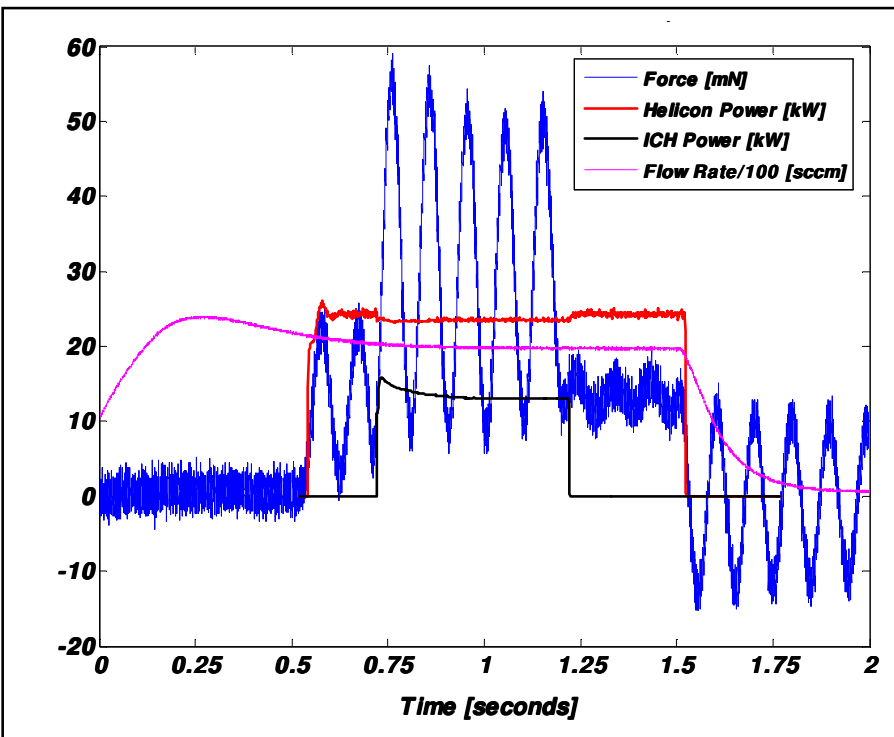

Figure 11. Force measurement for a VX-100 shot. 25 kW RF power into the helicon stage, $13 \mathrm{~kW} R F$ power into the ICRH stage, $2000 \mathrm{sccm}$ Ar.

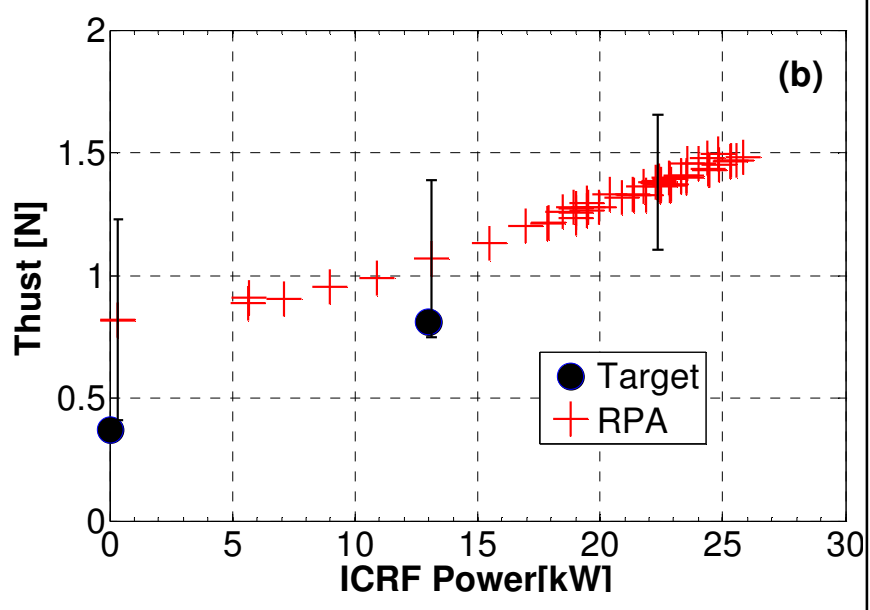

Figure 12. PMFS force data and RPA / ion flux force data for the VX-100. 
The overestimate of the force values from the RPA / ion flux method is identified as an error in estimate of the local plasma potential. If the conservative PMFS force data are used to scale up to $26 \mathrm{~kW}$ ICRH RF power, a $25 \mathrm{~kW}$ helicon plus $26 \mathrm{~kW}$ ICRH VX-100 shot produces over $1300 \mathrm{mN}$ of force.

\section{Momentum Flux Parameters}

\section{A. Surface Sputtering}

Target material choice for the PMFS is a nontrivial task owing to the bombardment from high energy ions in the exhaust plume of the HET. At a maximum operating power level, the P5 HET was able to produce incident ions with an average energy of approximately 440 $\mathrm{eV}$. This ion energy is far beyond the sputter threshold of any material. One simply tries to reduce the sputtering yield in order to reduce the associated momentum corrections and to reduce contamination of the HET from sputtered target material. With an $\mathrm{Ar}, \mathrm{Kr}$, or Xe propellant, the natural target material choice for low sputter yield is graphite. This low yield is in part a result of the covalent $\mathrm{C}-\mathrm{C}$ bond strength which results in the lowest sputter yield compared to any other material (with the exception of diamond) when the incident particles are more than twice as massive as the target atom. ${ }^{14}$ Experiments have also shown that for incident $\mathrm{Xe}$ ions, graphite has the lowest sputtering yield (with the exception of diamond). ${ }^{15} \quad$ In order to quantify sputtering yield rates in this experiment campaign, an RPA was used to measure the incident ion energy distribution from the HET for every force measurement data point. For typical operation, the P5 HET produces ions with an average energy equal to $50 \mathrm{~V}$ less than the anode voltage. That is, $\mathrm{Xe}$ ions with an average energy of $250 \mathrm{eV}$ are observed from an anode setting of $300 \mathrm{~V}$ with the P5 HET. ${ }^{11}$

The sputtering yield from a target surface is also highly dependent on the incident angle of the incoming particle, where incident particle trajectories that are normal to the surface generally produce lower sputtering yields and incident particle trajectories that are $\sim 70$ to 85 degrees from normal have the largest sputtering

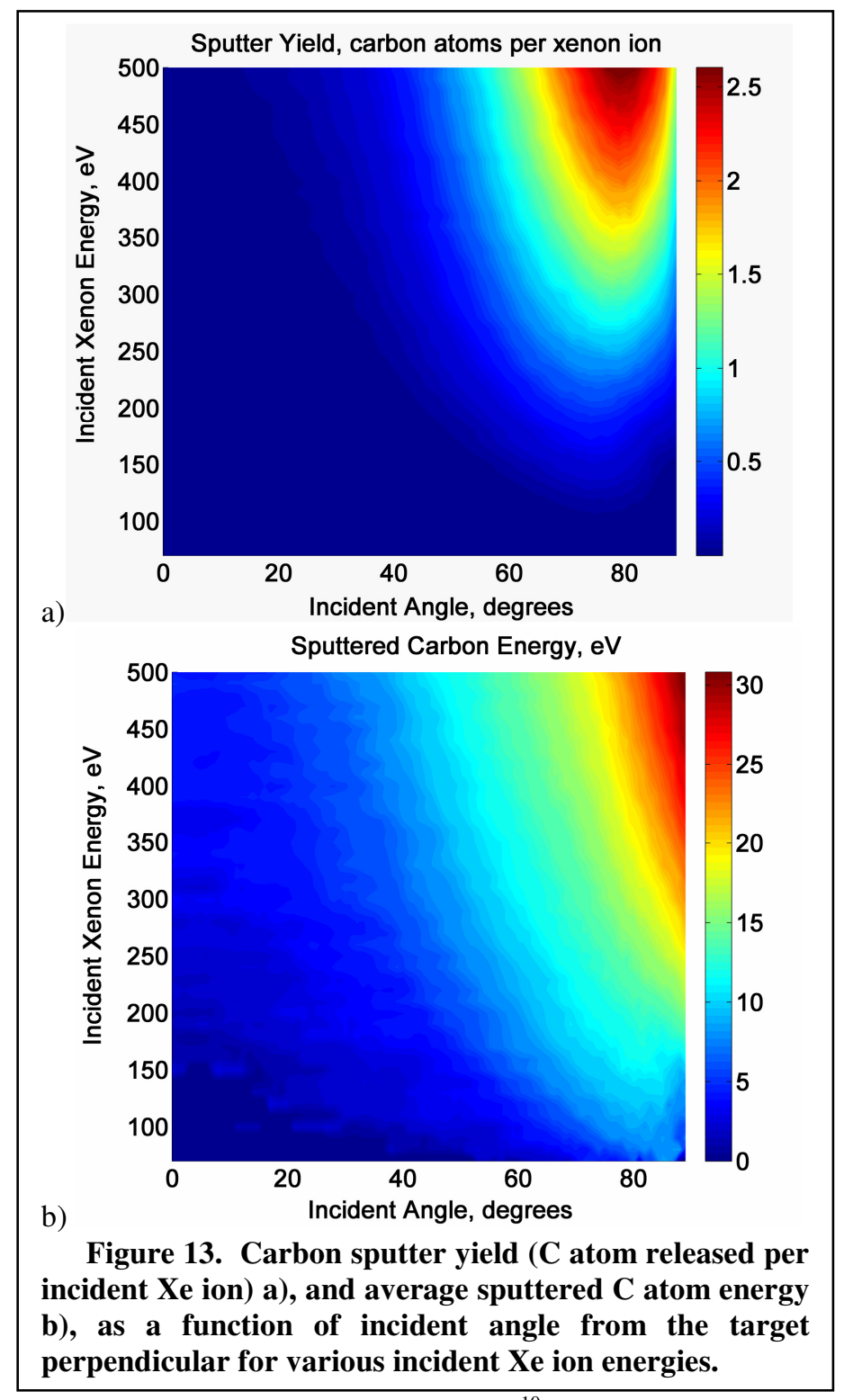
yields. Figure 13 a) and b) show the Stopping and Range of Ions in Matter (SRIM) code ${ }^{10}$ simulations for graphite sputter yield and energy per sputtered carbon atom as a function of incident angle from normal for a range of incident Xe ion energy.

In Fig. 14, a Xe particle with an incident angle of 0 degrees means the particle is perpendicular (normal) to the surface.

SRIM sputter yield and sputtered particle energy simulations were completed once the average incident $\mathrm{Xe}$ ion energy was determined from RPA data and the possible incident ion trajectories were determined from the target to thruster separation distance and thruster dimensions. The SRIM simulations gave the yield and energy of sputtered 
$\mathrm{C}$ atoms. These results were used to find the added momentum flux on the target caused by the exiting sputtered particles. The sputter simulation output is presented in Tables 3 and 4 for the separation distance experiment and the power variation experiment respectively. The last row in Tables 3 and 4 shows the momentum change on the PMFS force target that was caused by sputtered $\mathrm{C}$ atoms leaving the target surface.

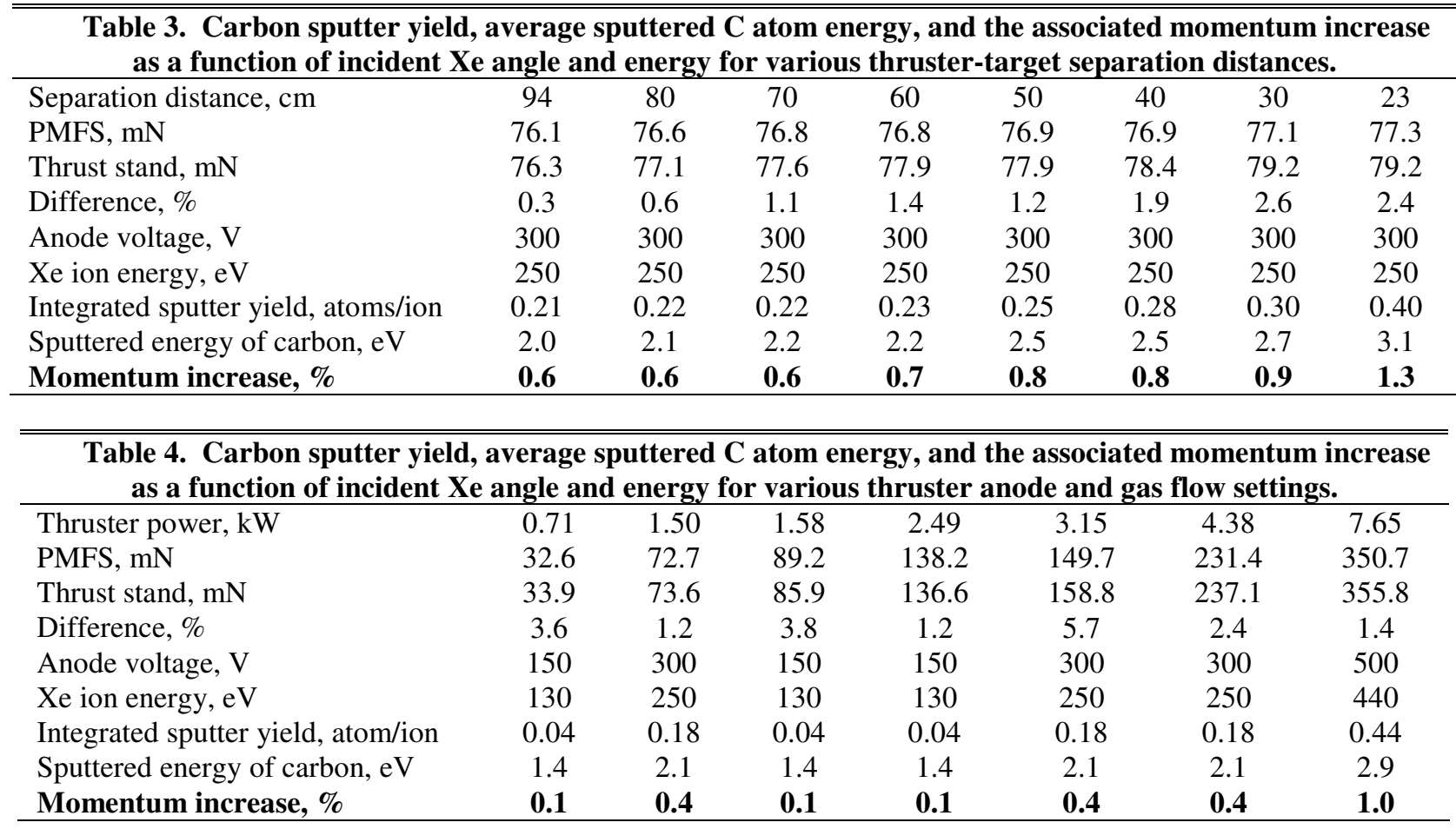

Because the sputter yield varies with incident particle angle, an integrated value was computed based on the probable incident ion trajectories from the HET channel impacting an annular section of the circular PMFS target. Ion energy values were measured with an RPA at the $53.5 \mathrm{~cm}$ location, and were used for all sputter calculations with the local ion flux measurements from each location. This is presented in Tables 4 and 5 as integrated sputter yield.

Simulations from SRIM show that the Xe ions with an energy between 8 and $500 \mathrm{eV}$ implant themselves 1 to 4 $\mathrm{nm}, 10$ to 40 times the atomic spacing for carbon atoms, into the surface of the graphite target in an inelastic collision and do not immediately 'bounce' off of the target surface in an elastic collision. For Xe particles impacting a graphite surface, these inelastic implantations occur for Xe particle energies above $\sim 8 \mathrm{eV}$, roughly the $\mathrm{C}$ - $\mathrm{C}$ surface binding energy for graphite. This inelastic ion implantation claim is supported by the experimental force comparison results in sections III A and C.

According to the SRIM simulations, the increase in measured momentum from carbon atoms sputtered off of the PMFS graphite target is at most $1.3 \%$ of the total measured momentum from the incident Xe ions. This small correction is included in all of the measurements presented in this paper, but is typically much less than the other errors in the measurements.

\section{B. Neutral Reflection and Charge Exchange}

A small fraction of the particles from the P5 HET hit the PMFS target as cold, unaccelerated, neutrals. A cold neutral particle would bounce off the target, thus contributing twice the amount of momentum that it carries towards the PMFS target. However, these cold gas particles carry negligible momentum compared to the accelerated ions and in this case, no neutral gas corrections were needed. Furthermore, the fraction of unaccelerated neutrals to accelerated ions is approximately $20 \% .{ }^{16}$ A more serious concern is hot neutrals created from fast ions undergoing charge exchange with the atoms of an ambient neutral gas population. Typically these energetic neutrals would go undetected by the Faraday probe that was used to detect the radial ion flux. However, as long as the charge exchange fraction is low compared to the ion population and/or the concentration of charge exchanged neutrals is proportional to the ion flux, charge exchanged neutrals do not significantly affect the accuracy of the PMFS device. 
As estimated by Randolph et al. ${ }^{13}$, only $2 \%$ of $200 \mathrm{eV}$ Xe ions undergo CEX within $100 \mathrm{~cm}$ with a background Xe pressure of $3 \times 10^{-6}$ Torr. With all of these factors combined, the cold gas contributes negligible momentum compared to the accelerated ions in the P5 HET at nominal operating conditions.

The Xe ions that become implanted within the graphite target may eventually make their way back to the front surface and escape from the target as neutral Xe atoms. However, the neutral Xe atoms would escape in an isotropic distribution as cold neutrals and also carry away negligible momentum compared to the high energy incident Xe ions.

The effect of charge-exchange (CEX) particles and doubly-charged ions is also negligible as long as the CEX and doubly-ionized fluxes are directly proportional to the ion flux, or the fraction of these CEX neutrals and doublycharged ions is small compared to singly-charged ions. This is a reasonable assumption based on previous data taken with the P5 HET. ${ }^{17}$

\section{Conclusion}

The Plasma Momentum Flux Sensor (PMFS) was shown to agree well with an inverted pendulum thrust stand, with a maximum observed difference of approximately $6 \%$ and an average difference of approximately $2 \%$ over a large range of force, ion energy, and neutral gas flow settings. It is often cumbersome or impossible to mount heavy thrusters on pendulum-type thrust stands. Likewise, the cost associated with designing an appropriate thrust stand can be prohibitive to some research efforts. As a viable alternative, the PMFS can be used as a highly accurate force sensor for a large range of flowing plasma conditions. Because the PMFS has demonstrated its accuracy as a plasma force diagnostic and is a simple, robust, and low-cost device, it should find considerable application in the flowing plasma and electric propulsion communities. The PMFS was used with the Variable Specific Impulse Magnetoplasma Rocket (VASIMR) and data with the VX-100 indicate a force on the PMFS target of greater than $810 \mathrm{mN}$ for a $25 \mathrm{~kW}$ helicon plus $13 \mathrm{~kW}$ ICRH pulse, and over $1300 \mathrm{mN}$ for a $25 \mathrm{~kW}$ helicon plus $25 \mathrm{~kW}$ ICRH pulse.

\section{Acknowledgments}

The authors would like to thank the University of Houston Institute for Space Systems Operations (ISSO) postdoctoral fellowship program, and the Ad Astra Rocket Company for travel and experiment support.

\section{References}

1 Chavers, D.G., Chang-Diaz, F.R., "Momentum Flux Measuring Instrument for Neutral and Charged Particle Flows," Rev. Sci. Instrum., Vol. 73, No. 10, 2002.

2 Cohen, S.A. Zonca, F., Timberlake, J., Bennett, T., Cuthbertson, J., Langer, W., Motley, R., “An Instrument for Measuring the Momentum Flux From Atomic and Charged Particle Jets,” Rev. Sci. Instrum. 61, 3586, 1990.

3 Nedzelskiy, I.S., Silva, C., Fernandes, H., Duarte, P., Varandas, C.A.F, "Compact Cantilever Force Probe for Plasma Pressure Measurements," Rev. Sci. Instrum. 78, 123505, 2007.

4 Yeha, F.B., Wei, P.S., "The Effect of Sheath on Plasma Momentum Transport to an Electrically Biased Surface," International Journal of Heat and Mass Transfer, Vol. 48, Issue 11, pp. 2198-2208, May 2005.

5 R.Y. Gnizdor, et al, "Research of the dynamic pressure distribution in the plume of the SPT," IEPC Paper 1997-153, International Electric Propulsion Conference, Cleveland, Ohio, 24-28 Aug 1997.

6 Haas, J.M., "Low-perturbation Interrogation of the Internal and Near-field Plasma Structure of a Hall Thruster Using a High-Speed Probe Positioning System," Thesis, Dept. of Aerospace Engineering, University of Michigan, 2001.

7 Haas, J.M., Gallimore, A.D., "An Investigation of Internal Ion Number Density and Electron Temperature Profiles in a Laboratory-Model Hall Thruster," AIAA Paper 2000-3422, 36th AIAA/ASME/SAE/ASEE Joint Propulsion Conference, Huntsville, AL,16-19 July, 2000.

8 Haas, J.M. Gulczinski III, F.S., Gallimore, A.D., Spanjers, G.G., Spores, R.A, "Performance characteristics of a 5 kW laboratory Hall thruster," AIAA Paper 1998-3503, 34th AIAA/ASME/SAE/ASEE Joint Propulsion Conference, Cleveland, OH,12-15 July 1998. 
9 Hofer, R.R., "Development and Characterization of High-Efficiency, High-Specific Impulse Xenon Hall Thrusters," Ph.D. Dissertation, University of Michigan, 2004.

10 Haag, T.W., "Design of a Thrust Stand for High Power Electric Propulsion Devices," AIAA Paper 1989-2829, 25th AIAA/ASME/SAE/ASEE Joint Propulsion Conference, Monterey, CA, 10-13Jul. 1989.

11 Gulczinksi, F.S., Hofer, R.R., and Gallimore A.D., "Near-field Ion Energy and Species Measurements of a $5 \mathrm{~kW}$ Laboratory Hall Thruster, " AIAA Paper 1999-2430, 35th AIAA/ASME/SAE/ASEE Joint Propulsion Conference, Los Angeles, CA, 20-23June 1999.

12 Ziegler, J.F., Biersack,J.P., SRIM: The Stopping and Range of Ions in Matter, Software Package, SRIM version 2008.

13 Randolph, T., Kim, V., Kaufman, H. R., Kozubsky, K., Zhurin, V. V., Day, M. "Facility Effects on Stationary Plasma Thruster Testing," IEPC Paper 1993-093, International Electric Propulsion Conference, Seattle, WA, 13-16 Sept. 1993.

14 Stangeby, P.C., “The Plasma Boundary of Magnetic Fusion Devices,” Institute of Physics, Bristol, pp. 46, 113, and 647, 2000 ,

15 Doerner, R.P., Whyte, D.G., Goebel, D.M., "Sputtering Yield Measurements During Low Energy Xenon Plasma Bombardment,” J. Appl. Phys. 93, 5816, 2003.

16 Hofer, R.R., Jankovsky, R.S., Gallimore, A.D., "High-Specific Impulse Hall Thrusters, Part 2: Efficiency Analysis," AIAA Journal of Propulsion and Power, 22, 4, 732-740, 2006.

17 Azziz, Y., "Experimental and Theoretical Characterization of a Hall Thruster Plume," Ph.D. Dissertation, Aeronautics and Astronautics Dept., Massachusetts Institute of Technology, Cambridge, MA, 2007. 